\title{
Robust Photometric Invariant Features from the
}

\section{Color Tensor}

\author{
J. van de Weijer, Th. Gevers and A.W.M. Smeulders
}

\begin{abstract}
Luminance-based features are widely used as low-level input for computer vision applications, even when color data is available. Extension of feature detection to the color domain prevents information loss due to isoluminance and allows to exploit the photometric information. To fully exploit the extra information in the color data, the vector nature of color data has to be taken into account and a sound framework is needed to combine feature and photometric invariance theory.

In this paper we focus on the structure tensor, or color tensor, which adequately handles the vector nature of color images. Further, we combine the features based on the color tensor with photometric invariant derivatives to arrive at photometric invariant features. We circumvent the drawback of unstable photometric invariants by deriving an uncertainty measure to accompany the photometric invariant derivatives. The uncertainty is incorporated in the color tensor, hereby allowing the computation of robust photometric invariant features. The combination of the photometric invariance theory and tensorbased features allows for detection of a variety of features such as photometric invariant edges, corners, optical flow and curvature. The proposed features are tested for noise characteristics and robustness to photometric changes. Experiments show that the proposed features are robust to scene incidental events and that the proposed uncertainty measure improves the applicability of full invariants.
\end{abstract}

\section{INTRODUCTION}

Differential-based features such as edges, corners, and salient points, are used abundantly in a variety of applications such as matching, object recognition, and object tracking [13], [22], [24]. We differ between feature detection and feature extraction. Feature detection aims at finding the position of features in the images. Whereas for feature extraction, a position in the images is described by a set of features, which characterize the local neighborhood. Although the majority of images is recorded in color format nowadays, computer vision research is still mostly restricted 
restricted to luminance-based feature detection and extraction. In this paper we focus on color information to detect and extract features.

In the basic approach to color images the gradient is computed from the derivatives of the separate channels. The derivatives of a single edge can point in opposing directions for the separate channels. DiZenzo [5] argues that a simple summation of the derivatives ignores the correlation between the channels. This also happens by converting the color image to luminance values. In the case of isoluminance of adjacent color regions it will lead to cancellation of the edge. As a solution to the opposing vector problem, DiZenzo proposes the color tensor for color gradient computation.

The same problem as occurs for color image derivatives, exists for oriented patterns (e.g. fingerprint images). Due to the high frequency nature of oriented patterns opposing derivative vectors occur in a small neighborhood. The same solution which was found for color image features, is used to compute features for oriented patterns. Kass and Witkin [16] derived orientation estimation from the structure tensor. Adaptations of the tensor lead to a variety of features, such as circle detectors and curvature estimation [3], [4], [12], [27]. Lee and Medioni [19] apply the structure tensor within the context of perceptual grouping.

A step forward in the understanding of color images was made by the dichromatic reflection model by Shafer [23]. The article provides a physical model which identifies how photometric changes, such as shadows and specularities, affect the $R G B$-values. On the basis of this model, others provided algorithms invariant to various photometric events such as shadows and specularities [8], [17]. The extension to differential photometric invariance was investigated by Geusebroek et al. [7]. Recently, van de Weijer et al. [26] introduced the photometric quasi-invariants which are a set of photometric invariant derivatives with better noise and stability characteristics compared to existing photometric invariants. Combining photometric quasi-invariants with derivative based feature detectors leads to features which can identify various physical causes, e.g. shadow corners and object corners. A drawback of the quasi-invariants is that they can only be applied for feature detection. In the case of feature extraction, where the values of multiple frames are compared, full invariance is necessary.

We propose a framework to combine the differential based-features with the photometric invariance theory. The framework is designed according to the following criteria: 1 . features must target the photometric variation needed for their application. To achieve that accidental physical 
events, such as shadows and specularities, will not influence results. 2. features must be robust against noise and should not contain instabilities. Especially for the photometric invariant features instabilities must be dissolved. 3. physically meaningful features should be independent of the accidental choice of the color coordinate frame. Next to satisfying the criteria the framework should also be generally applicable to existing features.

To meet these criteria we start from the observation that tensors are well-suited to combine first order derivatives for color images. The first contribution is a novel framework that combines tensor-based features with photometric derivatives for photometric invariant feature detection and extraction. The second contribution is that for feature extraction applications, for which quasiinvariants are unsuited, we propose a new uncertainty measure which robustifies the feature extraction. The third contribution is that the proposed features are proven to be invariant with respect to color coordinate transformations.

The paper is organized as follows. In section 2, the prerequisites for color feature detection from tensors are discussed. In section 3, an uncertainty measure is proposed. Based on this uncertainty measure robust photometric feature extraction is derived. In section 4, a overview of tensor-based features is given. Section 5, provides several experiments and section 6 contains the concluding remarks.

\section{TENSOR-BASED FEATURES FOR COLOR IMAGES}

The extension of differential-based operations to color images can be done in various ways. The main challenge to color feature detection is how to transform the $3 \mathrm{D}$-color differential structure to a representation of the presence of a feature. In this section we ensure that the transformation agrees with the criteria mentioned in the introduction. In section II-A the invariance with respect to color coordinate transformation is discussed. In section II-B the transformation is written in tensor mathematics which links it with a set of tensor based features, thereby ensuring generality. In section II-C the photometric invariance of the transformation is discussed.

\section{A. Invariance to Color Coordinate Transformations}

From a physical point of view only features make sense which are invariant to rotation of the coordinate axes. This starting point has been applied in the design of image geometry features, resulting in, for example, gradient and Laplace operators [6]. For the design of physically 


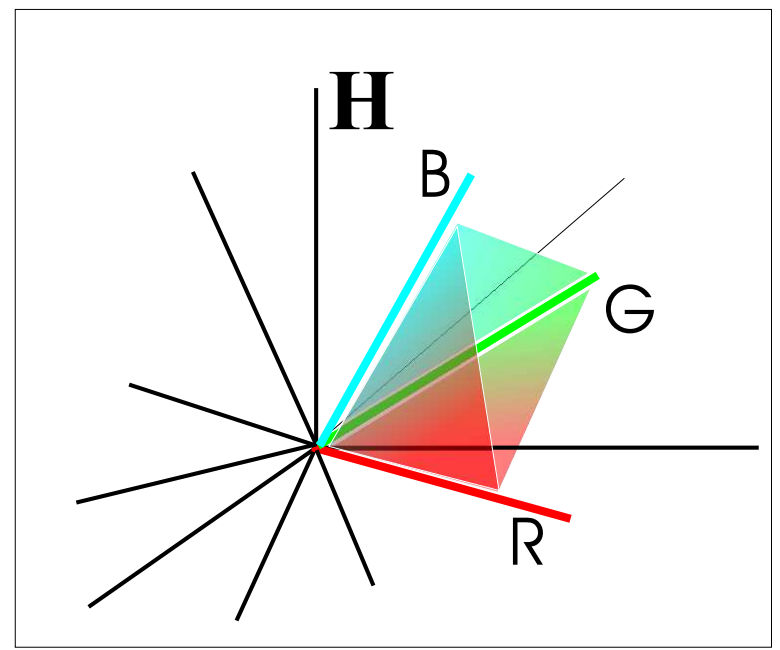

(a)

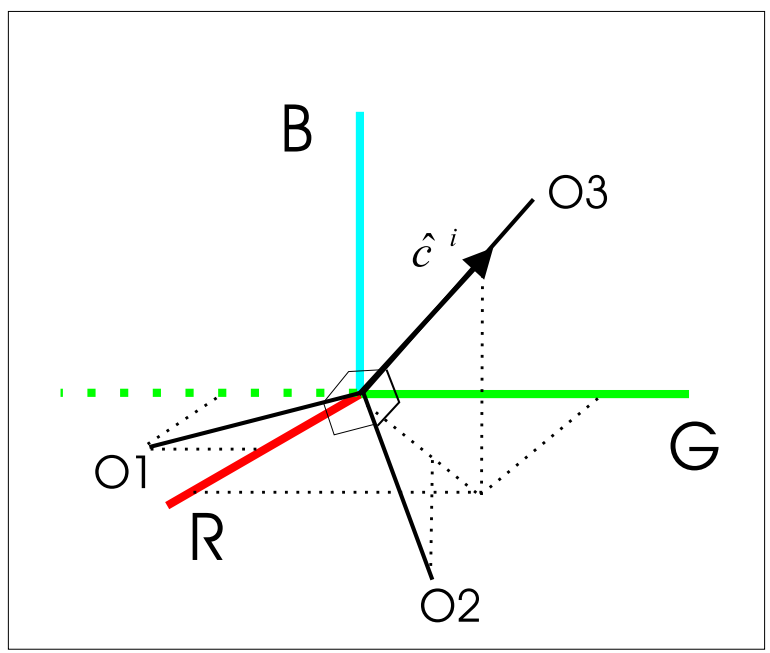

(b)

Fig. 1. a) The subspace of measured light in the Hilbert space of possible spectra. b) The RGB coordinate system and an alternative orthonormal color coordinate system which spans the same subspace.

meaningful color features not only the invariance with respect to spatial coordinate changes is desired but also the invariance with respect to color coordinate systems rotations. Features based on different measurement devices which measure the same spectral space should yield the same results.

For color images, values are represented in the $R G B$ coordinate system. In fact, the $\infty$ dimensional Hilbert space is sampled with three probes which results in the red, green and blue channels (see Fig. 1). For operations on the color coordinate system to be physically meaningful they should be independent of orthonormal transformation of the three axes in Hilbert space. As an example of an orthonormal color coordinate system the opponent color space can be taken (see Fig. 1b). The opponent color space spans the same subspace as the subspace defined by the $R G B$-axes and hence both subspaces should yield the same features.

\section{B. The Color Tensor}

Simply summing differential structure of various color channels may result in cancellation even when evident structure exists in the image [5]. Rather than adding the direction information, (defined on $[0,2 \pi\rangle$,) of the channels, it is more appropriate to sum the orientation information (, defined on $[0, \pi\rangle$ ). Such a method is provided by tensor mathematics for which vectors in 
opposite directions reinforce one another. Tensors describe the local orientation rather than the direction. More precisely, the tensor of a vector and its $180^{\circ}$ rotated counterpart vector are equal. It is for that reason that we use the tensor as a basis for color feature detection.

Given an image $f$, the structure tensor is given by [4]

$$
\mathbf{G}=\left(\begin{array}{cc}
\overline{f_{x}^{2}} & \overline{f_{x} f_{y}} \\
\overline{f_{x} f_{y}} & \overline{f_{y}^{2}}
\end{array}\right),
$$

where the subscripts indicate spatial derivatives and the bar - indicates convolution with a Gaussian filter. Note that there are two scales involved in the computation of the structure tensor. Firstly, the scale at which the derivatives are computed and secondly the tensor-scale which is the scale at which the spatial derivatives are averaged. The structure tensor describes the local differential structure of images, and is suited to find features such as edges and corners [3], [5], [12]. For a multichannel image $\mathbf{f}=\left(f^{1}, f^{2}, \ldots, f^{n}\right)^{T}$, the structure tensor is given by

$$
\mathbf{G}=\left(\begin{array}{cc}
\overline{\mathbf{f}_{x} \cdot \mathbf{f}_{x}} & \overline{\mathbf{f}_{x} \cdot \mathbf{f}_{y}} \\
\overline{\mathbf{f}_{y} \cdot \mathbf{f}_{x}} & \overline{\mathbf{f}_{y} \cdot \mathbf{f}_{y}}
\end{array}\right)
$$

In the case that $\mathbf{f}=(R, G, B)$, Eq. 2 is the color tensor. For derivatives which are accompanied with a weighting function, $w_{x}$ and $w_{y}$, which appoint a weight to every measurement in $\mathbf{f}_{\mathbf{x}}$ and $\mathbf{f}_{\mathbf{y}}$, the structure tensor is defined by

$$
\mathbf{G}=\left(\begin{array}{cc}
\frac{\overline{w_{x}^{2} \mathbf{f}_{x} \cdot \mathbf{f}_{x}}}{\overline{w_{x}^{2}}} & \frac{\overline{w_{x} w_{y} \mathbf{f}_{x} \cdot \mathbf{f}_{y}}}{\overline{w_{x} w_{y}}} \\
\frac{w_{y} w_{x} \mathbf{f}_{y} \cdot \mathbf{f}_{x}}{\overline{w_{y} w_{x}}} & \frac{w_{y}^{2} \mathbf{f}_{y} \cdot \mathbf{f}_{y}}{\overline{w_{y}^{2}}}
\end{array}\right) .
$$

In section II-A, we discussed that physically meaningful features should be invariant with respect to rotation of the color coordinates axes. The elements of the tensor are known to be invariant under rotation and translation of the spatial axes. To prove the invariant, we use the fact that $\frac{\partial}{\partial x} \mathbf{R} \mathbf{f}=\mathbf{R f}_{x}$, where $\mathbf{R}$ is a rotation operator,

$$
\overline{\left(\mathbf{R f}_{x}\right)^{T} \mathbf{R f}_{y}}=\overline{\mathbf{f}_{x}^{T} \mathbf{R}^{T} \mathbf{R} \mathbf{f}_{y}}=\overline{\mathbf{f}_{x}^{T} \mathbf{f}_{y}} .
$$

We rewritten the inner product with $\mathbf{f} \cdot \mathbf{f}=\mathbf{f}^{T} \mathbf{f}$

\section{Photometric Invariant Derivatives}

A good motivation for using color images is that photometric information can be exploited to understand the physical nature of features. For example, pixels can be classified as being from 
the same color but having different intensities which is possibly caused by a shadow or shading change in the image. Further, pixels differences can also indicate specular reflection. For many applications it is important to distinguish the scene incidental information from material edges. When color images are converted to luminance this photometric information is lost [9].

The incorporation of photometric invariance in Eq. 2 can be obtained by using invariant derivatives to compute the structure tensor. In [26] we derive photometric quasi-invariant derivatives and full invariant derivatives. Quasi-invariants differ from full invariants by the fact that they are variant with respect to a physical parameter. Full invariants can be computed from quasi-invariants by the normalization with a signal dependent scalar. The quasi-invariants have the advantage that they do not exhibit the instabilities common to full photometric invariants. However the applicability of the quasi-invariants is restricted to photometric invariant feature detection. For feature extraction full photometric invariance is desired.

The dichromatic model divides the reflection in the interface (specular) and body (diffuse) reflection component for optically inhomogeneous materials [23]. We assume white illumination, i.e. smooth spectrum of nearly equal energy at all wavelengths, and neutral interface reflection. For the validity of the photometric assumptions see [7], [23]. The RGB vector, $\mathbf{f}=(R, G, B)^{T}$, can be seen as a weighted summation of two vectors,

$$
\mathbf{f}=e\left(m^{b} \mathbf{c}^{b}+m^{i} \mathbf{c}^{i}\right)
$$

in which $\mathbf{c}^{b}$ is the color of the body reflectance, $\mathbf{c}^{i}$ the color of the interface reflectance (i.e. specularities or highlights), $m^{b}$ and $m^{i}$ are scalars representing the corresponding magnitudes of reflection and $e$ is the intensity of the light source. For matte surfaces there is no interface reflection and the model further simplifies to

$$
\mathbf{f}=e m^{b} \mathbf{c}^{b}
$$

The photometric derivative structure of the image can be computed by computing the spatial derivative of Eq. 5

$$
\mathbf{f}_{x}=e m^{b} \mathbf{c}_{x}^{b}+\left(e_{x} m^{b}+e m_{x}^{b}\right) \mathbf{c}^{b}+\left(e m_{x}^{i}+e_{x} m^{i}\right) \mathbf{c}^{i}
$$

The spatial derivative is a summation of three weighted vectors, successively caused by body reflectance, shading-shadow and specular changes. From Eq. 6 it follows that for matte surfaces 
the shadow-shading direction is parallel to the $R G B$ vector, $\mathbf{f} / / \mathbf{c}_{b}$. The specular direction follows from the assumption that the color of the light source is known.

For matte surfaces (i.e. $m^{i}=0$ ), the projection of the spatial derivative on the shadowshading axis yields the shadow-shading variant containing all energy which could be explained by changes due to shadow and shading. Subtraction of the shadow-shading variant from the total derivative results in the shadow-shading quasi-invariant

$$
\begin{aligned}
& \mathbf{S}_{x}=\left(\mathbf{f}_{x} \cdot \hat{\mathbf{f}}\right) \hat{\mathbf{f}}=\left(e m^{b}\left(\mathbf{c}_{x}^{b} \cdot \hat{\mathbf{f}}\right)+\left(e_{x} m^{b}+e m_{x}^{b}\right)\left|\mathbf{c}^{b}\right|\right) \hat{\mathbf{f}} \\
& \mathbf{S}_{\mathbf{x}}^{\mathbf{c}}=\mathbf{f}_{x}-\mathbf{S}_{x}=e m^{b}\left(\mathbf{c}_{x}^{b}-\left(\mathbf{c}_{x}^{b} \cdot \hat{\mathbf{f}}\right) \hat{\mathbf{f}}\right)
\end{aligned}
$$

which does not contain derivative energy caused by shadows and shading. The hat, ., is used to denote unit vectors. The full shadow-shading invariant results from normalizing the quasiinvariant by the intensity magnitude,

$$
\mathbf{s}_{x}=\frac{\mathbf{S}_{x}^{c}}{|\mathbf{f}|}=\frac{e m^{b}}{e m^{b}\left|\mathbf{c}^{b}\right|}\left(\mathbf{c}_{x}^{b}-\left(\mathbf{c}_{x}^{b}\right) \cdot \hat{\mathbf{f}}\right),
$$

which is invariant for $m^{b}$.

For the construction of the shadow-shading-specular quasi-invariant, we introduce the huedirection which is perpendicular to the light source direction $\hat{\mathbf{c}}^{i}$ and the shadow-shading direction $\hat{\mathbf{f}}$

$$
\hat{\mathbf{b}}=\frac{\hat{\mathbf{f}} \times \hat{\mathbf{c}}^{i}}{\left|\mathbf{f} \times \mathbf{c}^{i}\right|}
$$

Projection of the derivative, $\mathbf{f}_{x}$, on the hue direction results in the shadow-shading-specular quasi-invariant

$$
\mathbf{H}_{x}^{c}=\left(\mathbf{f}_{x} \cdot \hat{\mathbf{b}}\right) \hat{\mathbf{b}}=e m^{b}\left(\mathbf{c}_{x}^{b} \cdot \hat{\mathbf{b}}\right)+\left(e_{x} m^{b}+e m_{x}^{b}\right)\left(\mathbf{c}^{b} \cdot \mathbf{b}\right) .
$$

The second part of this equation is zero if we assume that shadow-shading changes do not occur within a specularity, since then either $\left(e_{x} m^{b}+e m_{x}^{b}\right)=0$ or $\left(\mathbf{c}^{\mathbf{b}} \cdot \mathbf{b}\right)=(\mathbf{f} \cdot \mathbf{b})=0$. Subtraction of the quasi-invariant from the spatial derivative results in the shadow-shading-specular variant

$$
\mathbf{H}_{x}=\mathbf{f}_{x}-\mathbf{H}_{x}^{c}
$$

The full shadow-shading invariant is computed by dividing the quasi-invariant by the saturation. The saturation is equal to the norm of the color-vector, $\mathbf{f}$, after the projection on the plane 
perpendicular to the light source direction (which is equal to subtraction of the part in the light source direction)

$$
\mathbf{h}_{x}=\frac{\mathbf{H}_{x}^{c}}{\left|\mathbf{f}-\left(\mathbf{f} \cdot \hat{\mathbf{c}}^{i}\right) \hat{\mathbf{c}}^{i}\right|}=\frac{e m^{b}}{e m^{b}\left|\mathbf{c}^{b}-\left(\mathbf{c}^{b} \cdot \hat{\mathbf{c}}^{i}\right) \hat{\mathbf{c}}^{i}\right|}\left(\mathbf{c}_{x}^{b} \cdot \hat{\mathbf{b}}\right) .
$$

This $\mathbf{h}_{x}$ is invariant for both $m^{i}$ and $m^{b}$.

By projecting the local spatial derivative on three photometric axis in the $R G B$ cube we have derived the photometric quasi-invariants. These can be combined with the structure tensor of Eq. 18 for photometric quasi-invariant feature detection. As discussed in section II-A we would like features to be independent of the accidental choice of the color coordinate frame. As a consequence a rotation of the color coordinates should result in the same rotation of the quasiinvariant derivatives. For example for the shadow-shading quasi-variant $\mathbf{S}_{x}$ this can be proven by

$$
\left(\left(\mathbf{R f}_{x}\right)^{T} \mathbf{R} \hat{\mathbf{f}}\right)(\mathbf{R} \hat{\mathbf{f}})=\left(\mathbf{f}_{x}^{T} \mathbf{R}^{T} \mathbf{R} \hat{\mathbf{f}}\right)(\mathbf{R} \hat{\mathbf{f}})=\mathbf{R}\left(\mathbf{f}_{x}^{T} \hat{\mathbf{f}}\right) \hat{\mathbf{f}}=\mathbf{R S}_{x}
$$

Similar proofs hold for the other photometric variants and quasi-invariants. The invariance with respect to color coordinate transformation of the shadow-shading full invariants follow from the fact that $|\mathbf{R f}|=|\mathbf{f}|$. For the shadow-shading-specular full-invariant, the invariance is proven by the fact that the inner product between two vectors remains the same under rotations, and therefore $\left|\mathbf{R f}-\left(\mathbf{R f} \cdot \mathbf{R} \hat{\mathbf{c}}^{i}\right) \mathbf{R} \hat{\mathbf{c}}^{i}\right|=\left|\mathbf{R}\left(\mathbf{f}-\left(\mathbf{f} \cdot \hat{\mathbf{c}}^{i}\right) \hat{\mathbf{c}}^{i}\right)\right|$. Since the elements of the structure tensor are also invariant for color coordinate transformations (see Eq 4) the combination of the quasiinvariants and the structure tensor into a quasi-invariant structure tensor is also invariant for color coordinate transformations.

\section{Robust Full Photometric InVARiAnCE}

In section II-C the quasi- and full invariant derivatives are described. The quasi-invariants outperform the full-invariants on discriminative power and are more robust to noise [26]. However, the quasi-invariants are not suited for applications which require feature extraction. These applications compare the photometric invariant values between various images and need full photometric invariance (see Table I). A disadvantage of full photometric invariants is that they are unstable in certain areas of the RGB-cube. E.g. the invariants for shadow-shading and specularities are unstable near the gray axis. These instabilities greatly reduce the applicability of the invariant derivatives for which a small deviation of the original pixel color value may 


\begin{tabular}{|l|c|c|}
\hline & detection & extraction \\
\hline quasi invariant & +++ & - \\
\hline full invariant & + & + \\
\hline robust full invariant & ++ & ++ \\
\hline
\end{tabular}

TABLE I

APPLICABILITY OF THE DIFFERENT INVARIANTS FOR FEATURE DETECTION AND EXTRACTION.

result in a large deviation of the invariant derivative. In this section, we propose a measure which describes the uncertainty of the photometric invariant derivatives, thereby allowing for robust full photometric invariant feature detection.

We will first derive the uncertainty for the shadow-shading full invariant from its relation to the quasi-invariant. We assume additive uncorrelated uniform Gaussian noise. Due to the highpass nature of differentiation we assume the noise of the zero order signal $(|\mathbf{f}|)$ to be negligible compared to the noise on the first order signal $\left(\mathbf{S}_{x}^{c}\right)$. In section II-C, the quasi-invariant has been derived by a linear projection of the derivative $\mathbf{f}_{x}$ on the plane perpendicular to the shadowshading direction. Therefore, uniform noise in $\mathbf{f}_{x}$ will result in uniform noise in $\mathbf{S}_{x}^{c}$. The noise in the full invariant can be written as

$$
\tilde{\mathbf{s}}_{x}=\frac{\mathbf{S}_{x}^{c}+\sigma}{|\mathbf{f}|}=\frac{\mathbf{S}_{x}^{c}}{|\mathbf{f}|}+\frac{\sigma}{|\mathbf{f}|} .
$$

The uncertainty of the measurement of $\tilde{\mathbf{s}}_{x}$ depends on the magnitude of $|\mathbf{f}|$. For small $|\mathbf{f}|$ the error increases proportionally. Therefore we propose to weight the full shadow-shading invariant with the function $w=|\mathbf{f}|$ to robustify the color tensor-based on the chromatic invariant. For shadow-shading invariance examples of the equations used to compute the color tensor are given in Table I.

For the shadow-shading-specular invariant, the weighting function should be proportional with the saturation, since

$$
\tilde{\mathbf{h}}_{x}=\frac{\mathbf{H}_{x}^{c}+\sigma}{|\mathbf{s}|}=\frac{\mathbf{H}_{x}^{c}}{|\mathbf{s}|}+\frac{\sigma}{|\mathbf{s}|} .
$$

This leads us to propose $w=|\mathbf{s}|$ as the weighting function of the hue derivative $\tilde{\mathbf{h}}_{x}$ see Fig. 2). On place where there is an edge, the saturation drops, and with the saturation the certainty of 


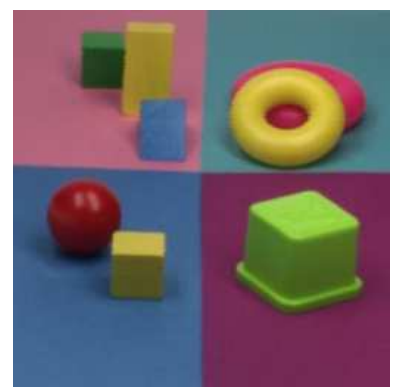

(a)

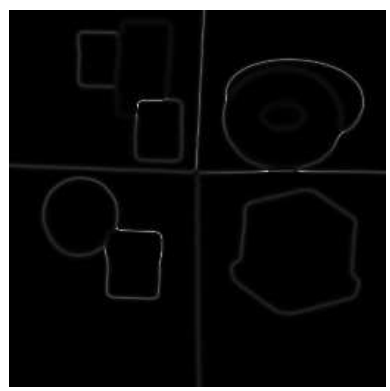

(b)

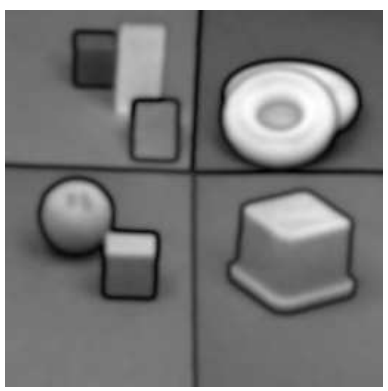

(c)

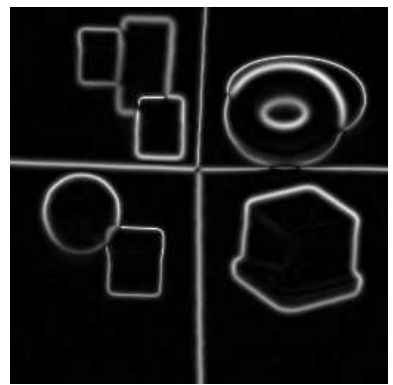

(d)

Fig. 2. a) test image b) hue derivative c) saturation d) quasi-invariant.

the hue measurement. The quasi-invariant (see Fig. 2d), which is equal to the weighted hue, is more stable than the full invariant derivative due to the incorporation of the certainty in the measurements. With the derived weighting function we can compute the robust photometric invariant tensor (Eq. 3).

The uncertainties of the full-invariant by ways of error-propagation have also been investigated by Gevers and Stokman [10]. Our assumption of uniform noise in the $R G B$ channels together with the choice of invariants based on orthogonal color space transformations leads to a simplification of the uncertainty measure. It also connects with the intuitive notion that the uncertainty of the hue is depended on the saturation and the uncertainty of the chromaticity (shadow-shading invariant) with the intensity.

\section{Color Tensor-BAsed FeAtures}

In this section we show the generality of the proposed method by summing features which can be derived from the color tensor. In section II-C and in section III we described how to compute invariant derivatives. Dependent on the task at hand we proposed to use either quasi-invariants for detection or robust full invariants for extraction. The features in this chapter will be derived for $\mathrm{g}_{x}$. By replacing the inner product of $\mathbf{g}_{x}$ by one of the following

$$
\left\{\overline{\mathbf{f}_{x} \cdot \mathbf{f}_{x}}, \overline{\mathbf{S}_{x}^{c} \cdot \mathbf{S}_{x}^{c}}, \frac{\overline{\mathbf{S}_{x}^{c} \cdot \mathbf{S}_{x}^{c}}}{\overline{|\mathbf{f}|^{2}}}, \overline{\mathbf{H}_{x}^{c} \cdot \mathbf{H}_{x}^{c}}, \frac{\overline{\mathbf{H}_{x}^{c} \cdot \mathbf{H}_{x}^{c}}}{\overline{|\mathbf{s}|^{2}}}\right\} .
$$

the acquired photometric invariant features are attained. In section IV-A we describe features derived from the eigenvalues of the tensor. In section IV-B features which are derived from an adapted version of the structure tensor and in section IV-C we describe color optical flow. 


\section{A. Eigenvalue-Based Features}

Eigenvalue analysis of the tensor leads to two eigenvalues which are defined by

$$
\begin{aligned}
& \lambda_{1}=\frac{1}{2}\left(\overline{\mathbf{g}_{x} \cdot \mathbf{g}_{x}}+\overline{\mathbf{g}_{y} \cdot \mathbf{g}_{y}}+\sqrt{\left(\overline{\mathbf{g}_{x} \cdot \mathbf{g}_{x}}-\overline{\mathbf{g}_{y} \cdot \mathbf{g}_{y}}\right)^{2}+\left(2 \overline{\mathbf{g}_{x} \cdot \mathbf{g}_{y}}\right)^{2}}\right) \\
& \lambda_{2}=\frac{1}{2}\left(\overline{\mathbf{g}_{x} \cdot \mathbf{g}_{x}}+\overline{\mathbf{g}_{y} \cdot \mathbf{g}_{y}}-\sqrt{\left(\overline{\mathbf{g}_{x} \cdot \mathbf{g}_{x}}-\overline{\mathbf{g}_{y} \cdot \mathbf{g}_{y}}\right)^{2}+\left(2 \overline{\mathbf{g}_{x} \cdot \mathbf{g}_{y}}\right)^{2}}\right)
\end{aligned}
$$

The direction of $\lambda_{1}$ indicates the prominent local orientation

$$
\theta=\frac{1}{2} \arctan \left(\frac{2 \overline{\mathbf{g}_{x} \cdot \mathbf{g}_{y}}}{\overline{\mathbf{g}_{x} \cdot \mathbf{g}_{x}}-\overline{\mathbf{g}_{y} \cdot \mathbf{g}_{y}}}\right) .
$$

The $\lambda$ 's can be combined to give the following local descriptors:

- $\lambda_{1}+\lambda_{2}$ describes the total local derivative energy.

- $\lambda_{1}$ is the derivative energy in the most prominent direction.

- $\lambda_{1}-\lambda_{2}$ describes the line-energy (see [21]). The derivative energy in the prominent orientation is corrected for the energy contributed by the noise $\lambda_{2}$.

- $\lambda_{2}$ describes the amount of derivative energy perpendicular to the prominent local orientation which is used to select features for tracking [24].

An often applied feature detector is the Harris corner detector [14]. The color Harris operator $H$ can be written as a function of the eigenvalues of the structure tensor

$$
\begin{aligned}
H \mathbf{f} & =\overline{\mathbf{g}_{x} \cdot \mathbf{g}_{x}} \overline{\mathbf{g}_{y} \cdot \mathbf{g}_{y}}-\overline{\mathbf{g}_{x} \cdot \mathbf{g}_{y}}-k\left(\overline{\mathbf{g}_{x} \cdot \mathbf{g}_{x}}+\overline{\mathbf{g}_{y} \cdot \mathbf{g}_{y}}\right)^{2} \\
& =\lambda_{1} \lambda_{2}-k\left(\lambda_{1}+\lambda_{2}\right)^{2} .
\end{aligned}
$$

\section{B. Adaptations of the Color Tensor}

The same equations as DiZenzo's equations for orientation estimation are found by Kass and Witkin [16]. They studied orientation estimation for oriented patterns (e.g. fingerprint images). Oriented patterns are defined as patterns with a dominant orientation everywhere. For oriented patterns other mathematics are needed than for regular object images. The local structure of object images is described by a step edge, whereas for oriented patterns the local structure is described as a set of lines (roof edges). Lines generate opposing vectors on a small scale. Hence for geometric operations on oriented patterns, methods are needed for which opposing vectors enforce one another. This is the same problem as encountered for all color images, where the opposing vector problem does not only occur for oriented patterns, but also for step edges, for which the opposing vectors occur in the different channels. Hence similar equations were found 
in both fields. Next to orientation estimation, a number of other estimators were proposed by oriented pattern research [12], [3], [27]. These operation are based on adaptations of the structure tensor and can also be applied to the color tensor.

The structure tensor of Eq. 2 can also be seen as a local projection of the derivative energy on two perpendicular axes, namely $\mathbf{u}_{1}=\left(\begin{array}{ll}1 & 0\end{array}\right)^{T}$ and $\mathbf{u}_{2}=\left(\begin{array}{ll}0 & 1\end{array}\right)^{T}$.

$$
\mathbf{G}^{\mathbf{u}_{1}, \mathbf{u}_{2}}=\left(\begin{array}{ll}
\overline{\left(\mathbf{G}_{x, y} \mathbf{u}_{1}\right) \cdot\left(\mathbf{G}_{x, y} \mathbf{u}_{1}\right)} & \overline{\left(\mathbf{G}_{x, y} \mathbf{u}_{1}\right) \cdot\left(\mathbf{G}_{x, y} \mathbf{u}_{2}\right)} \\
\overline{\left(\mathbf{G}_{x, y} \mathbf{u}_{1}\right) \cdot\left(\mathbf{G}_{x, y} \mathbf{u}_{2}\right)} & \overline{\left(\mathbf{G}_{x, y} \mathbf{u}_{2}\right) \cdot\left(\mathbf{G}_{x, y} \mathbf{u}_{2}\right)}
\end{array}\right)
$$

in which $\mathbf{G}_{x, y}=\left(\begin{array}{ll}\mathbf{g}_{x} & \mathbf{g}_{y}\end{array}\right)$. From the Lie group of transformation several other choices of perpendicular projections can be derived [3], [12]. They include feature extraction for circle, spiral and star-like structures.

The star and circle detector is given as an example. It is based on $\mathbf{u}_{1}=\frac{1}{\sqrt{x^{2}+y^{2}}}\left(\begin{array}{ll}x \quad y\end{array}\right)^{T}$

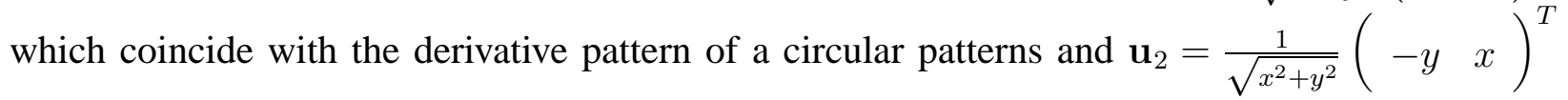
which denotes the perpendicular vector field which coincides with the derivative pattern of starlike patterns. These vectors can be used to compute the adapted structure tensor with Eq. 21. Only the elements on the diagonal have non zero entries and are equal to

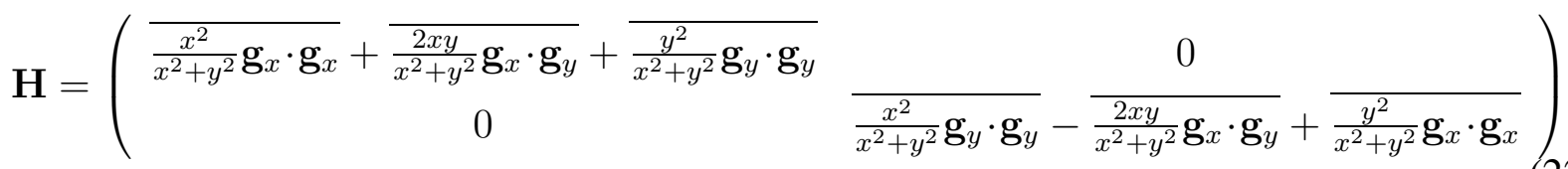

Here $\lambda_{1}$ describes the amount of derivative energy contributing to circular structures and $\lambda_{2}$ the derivative energy which describes a starlike structure. Similar to the proof given in Eq. 4 the elements of Eq. 22 can be proven to be invariant under transformations of the $R G B$-space.

Curvature is another feature which can be derived from an adaption of the structure tensor [27]. The fit between the local differential structure and a parabolic model function can be written as a function of the curvature. Finding the optimum of this function yields an estimation of the local curvature. For vector data the equation for the curvature is given by

$$
\kappa=\frac{\overline{w^{2} \mathbf{g}_{v} \cdot \mathbf{g}_{v}}-\overline{w^{2}} \cdot \overline{\mathbf{g}_{w} \cdot \mathbf{g}_{w}}-\sqrt{\left(\overline{w^{2}} \cdot \overline{\mathbf{g}_{w} \cdot \mathbf{g}_{w}}-\overline{w^{2} \mathbf{g}_{v} \cdot \mathbf{g}_{v}}\right)^{2}+4 \overline{w^{2}} \cdot \overline{w \mathbf{g}_{v} \cdot \mathbf{g}_{w}}}}{2 \overline{w^{2}} \cdot \overline{\mathbf{w}_{v} \cdot \mathbf{g}_{w}}}
$$

in which $\mathbf{f}_{v}$ and $\mathbf{f}_{w}$ are the derivatives in gauge coordinates. 


\section{Color Optical Flow}

Optical flow can also be computed from the structure tensor. This is originally proposed by Simoncelli [25] and has been extended to color in [2], [11]. The vector of a multi-channel point over time stays constant [15], [20]

$$
\frac{d \mathbf{g}}{d t}=\mathbf{0} .
$$

Differentiating yields the following set of equations

$$
\mathrm{G}_{\mathbf{x}, \mathbf{y}} \mathbf{v}+\mathrm{g}_{\mathrm{t}}=\mathbf{0}
$$

with $\mathbf{v}$ the optical flow. To solve the singularity problem and to robustify the optical flow computation we follow Simoncelli [25] and assume a constant flow within a Gaussian window. Solving Eq. 25 leads to the following optical flow equation

$$
\mathbf{v}=\overline{\left(\mathbf{G}_{x, y} \cdot \mathbf{G}_{x, y}\right)^{-1}} \overline{\mathbf{G}_{x, y} \cdot \mathbf{g}_{t}}=\mathbf{M}^{-1} \mathbf{b}
$$

where

$$
\mathbf{M}=\left(\begin{array}{ll}
\overline{\mathbf{g}_{x} \cdot \mathbf{g}_{x}} & \overline{\mathbf{g}_{x} \cdot \mathbf{g}_{y}} \\
\overline{\mathbf{g}_{y} \cdot \mathbf{g}_{x}} & \overline{\mathbf{g}_{y} \cdot \mathbf{g}_{y}}
\end{array}\right)
$$

and

$$
\mathbf{b}=\left(\begin{array}{l}
\overline{\mathbf{g}_{x} \cdot \mathbf{g}_{t}} \\
\overline{\mathbf{g}_{y} \cdot \mathbf{g}_{t}}
\end{array}\right) .
$$

The assumption of color optical flow based on $R G B$ is that $R G B$ pixel values remain constant over time (see Eq. 24). A change of brightness introduced due to a shadow, or a light source with fluctuating brightness such as the sun results in non existent optical flow. This problem can be overcome by assuming constant chromaticity over time. For photometric invariant optical flow, full invariance is necessary since the optical flow estimation is based upon comparing the (extracted) edge response of multiple frames. Consequently photometric invariant optical flow can be attained by replacing the inner product of $\mathrm{g}_{x}$ by one of the following

$$
\left\{\frac{\overline{\mathbf{S}_{x}^{c} \cdot \mathbf{S}_{x}^{c}}}{\overline{|\mathbf{f}|^{2}}}, \frac{\overline{\mathbf{H}_{x}^{c} \cdot \mathbf{H}_{x}^{c}}}{\overline{|\mathbf{s}|^{2}}}\right\} \text {. }
$$




\begin{tabular}{|l|c|c|c|c|}
\hline & \multicolumn{2}{c|}{$\begin{array}{c}\text { detection } \\
\text { error \% }\end{array}$} & \multicolumn{2}{c|}{$\begin{array}{c}\text { extraction } \\
\text { error \% }\end{array}$} \\
\hline \multicolumn{1}{|c|}{ std. noise } & 5 & 20 & 5 & 20 \\
\hline shadow-shading & & & & \\
quasi invariant & $\underline{5.1}$ & $\underline{20.2}$ & 100 & 100 \\
full invariant & 11.7 & 50.1 & 8.7 & 56.6 \\
robust full invariant & 6.4 & 37.7 & $\underline{3.0}$ & $\underline{35.3}$ \\
\hline shadow-shading-specular & & & & \\
quasi invariant & $\underline{9.7}$ & $\underline{46.6}$ & 100 & 98.2 \\
full invariant & 38.8 & 75.5 & 62.3 & 84.0 \\
robust full invariant & 15.7 & 60.2 & $\underline{9.8}$ & $\underline{66.6}$ \\
\hline
\end{tabular}

TABLE II

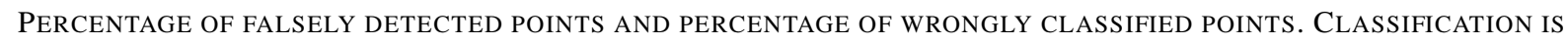
BASED ON THE EXTRACTION OF INVARIANT INFORMATION. UNCORRELATED GAUSSIAN NOISE IS ADDED WITH STANDARD DEVIATION 5 AND 20.

\section{EXPERIMENTS}

The experiments test the features on the required criteria of our framework: 1. photometric invariance, 2. robustness. The third criterium, i.e. invariance with respect to color coordinate transformations, we have already proven theoretically. In this section we aim to demonstrate invariance by experiment and illustrate the generality of the experiments by the variety of examples. For all experiments the derivatives are computed with a Gaussian derivative of $\sigma=1$ and the color tensor scale is computed with $\sigma=3$, except when mentioned otherwise. The experiments are performed using a Sony 3CCD color camera XC-003P, Matrox Corona Framegrabber, and two Osram 18 Watt "Lumilux deLuxe daylight" fluorescent light sources.

\section{A. Photometric Invariant Harris Point Detection}

Robustness with respect to photometric changes, stability of the invariants, and robustness to noise, are tested. Further the ability of invariants to detect and extract features is examined, see also Table I. The experiment is performed with the photometric invariant Harris corner detector (Eq. 20) and is executed on the Soil47 multi object set [18], which comprises of 23 images, see 


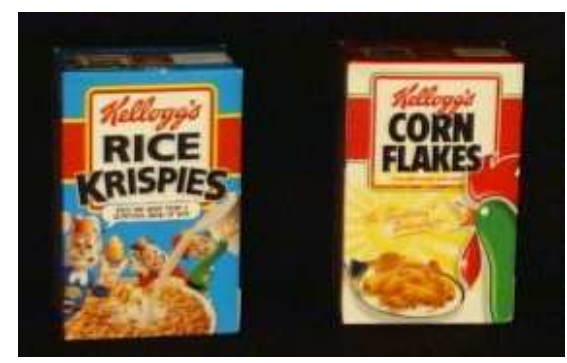

(a)

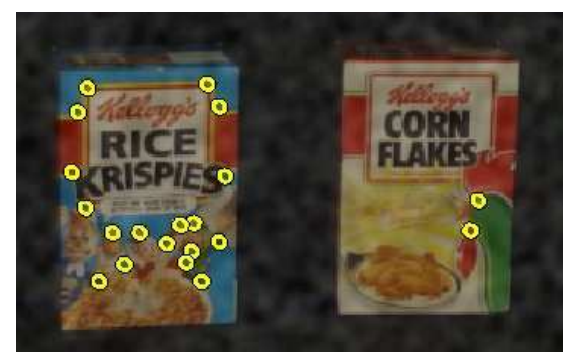

(b)

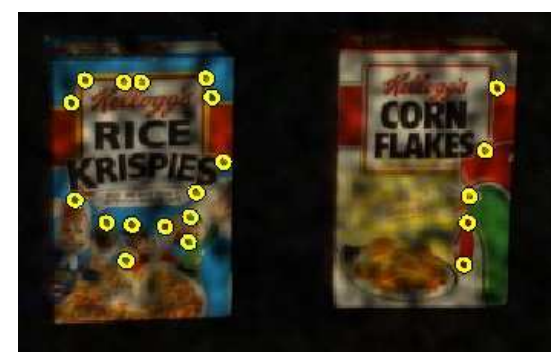

(c)

Fig. 3. a) An example from Soil-47 image. b) shadow-shading distortion with the shadow-shading quasi-invariant Harris points superimposed c) specular distortion and the shadow-shading-specular Harris points superimposed.

Fig. 3a.

First, the feature detection accuracy of the invariants is tested. For each image and invariant, the 20 most prominent Harris points are extracted. Next, Gaussian uncorrelated noise is added to the data, and the Harris point detection is computed 10 times per image. The percentage of points which do not correspond to the Harris points in the noise-less case are given in Table II. The Harris point detector based on the quasi-invariant outperforms the alternatives. The instability within the full invariant can be partially repaired by the robust full invariant, however for detection purposes the quasi-invariants remain the best choice.

Next, the feature extraction for the invariants is tested. Again the 20 most prominent Harris points are detected in the noise free image. For these points the photometric invariant derivative energy is extracted by $\sqrt{\lambda_{1}+\lambda_{2}-2 \lambda_{n}}$. Where $\lambda_{n}$ is an estimation of the noise which contributes to the energy in both $\lambda_{1}$ and $\lambda_{2}$. To imitate photometric variations of images we apply the following photometric distortion to the images (compare with Eq. 5)

$$
\mathbf{g}(\mathbf{x})=\alpha(\mathbf{x}) \mathbf{f}(\mathbf{x})+\beta(\mathbf{x}) \mathbf{c}^{i}+\eta(\mathbf{x})
$$

Where $\alpha(\mathbf{x})$ is a smooth function resembling variation similar to shading and shadow effects, $\beta(\mathbf{x})$ is a smooth function which imitates specular reflections, and $\eta(\mathbf{x})$ is Gaussian noise. To test the shadow-shading extraction $\alpha(\mathbf{x})$ is chosen to vary between 0 and 1 , and $\beta(\mathbf{x})$ is 0 . To test the shadow-shading-specular invariants $\alpha(\mathbf{x})$ was chosen constant at 0.7 and $\beta(\mathbf{x})$ varied between zero and fifty. After the photometric distortion the derivative energy is extracted at the same twenty points. The extraction is considered correct if the deviation of the derivative energy 


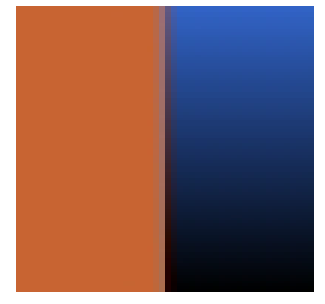

(a)

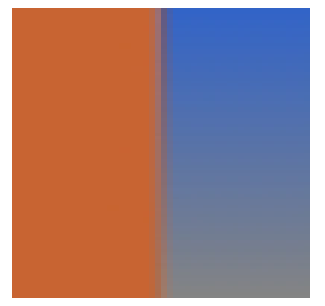

(d)

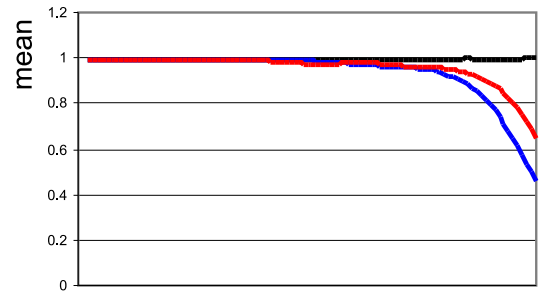

(b)

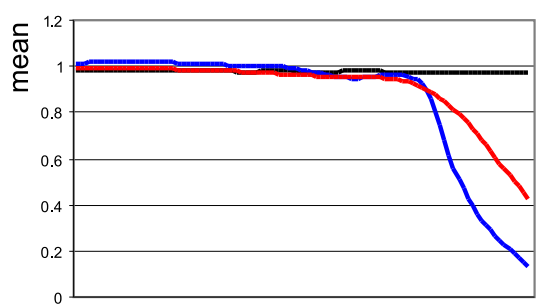

(e)

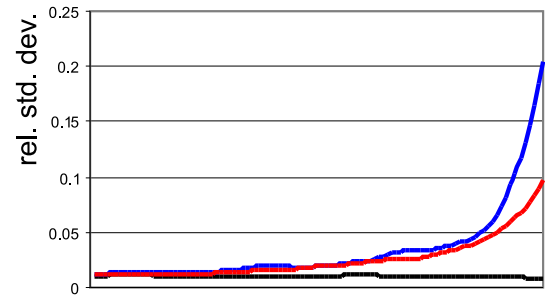

(c)

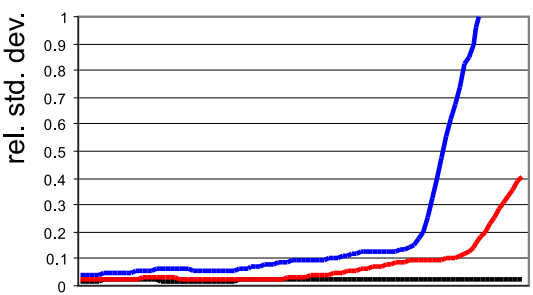

(f) achromaticity

Fig. 4. a,d) frame from test sequence with constant optical fbw of one pixel per frame. b,c) mean and relative standard deviation mean of the optical fbw based on $R G B$ (black line), shadow-shading invariant (blue line) and robust shadow-shading invariant (red line). e,f) mean and relative standard deviation of the optical fbw based on $R G B$ (black line), shadow-shading-specular invariant (blue line) and robust shadow-shading-specular invariant (red line).

between the distorted and the noise-free case is less then 10 percent. The results are given in Table II. Quasi-invariants which not suited for extraction have a hundred percent error. The full invariants have better results but with worsening signal-to-noise ratio its performance drops drastically. In accordance with the theory in section III the robust full invariants successfully improve the performance.

\section{B. Color Optical Flow}

Robustness of the full photometric invariance features is tested on photometric invariant optical flow estimation. The optical flow is estimated on a synthetical image sequence with constant optical flow. We use the robust full photometric structure tensor for the estimation of optical flow and compare it with 'classical' photometric optical flow as proposed by [11]. Derivatives are computed with a Gaussian derivative of $\sigma=1$ and the color tensor scale is $\sigma=5$.

The shadow-shading photometric optical flow is tested on image with decreasing intensity (see Fig. 4a) ) which is shifted one pixel per frame. Uncorrelated Gaussian noise with $\sigma=20$ is added to the sequence. In Fig. 4b,c the mean and the standard deviation of the optical flow along the 


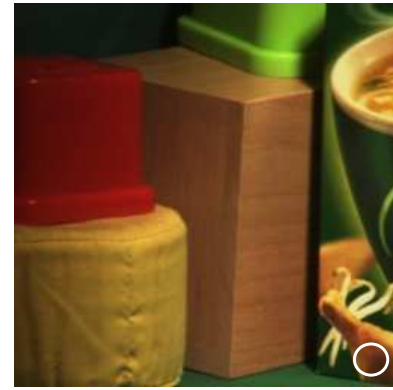

(a)

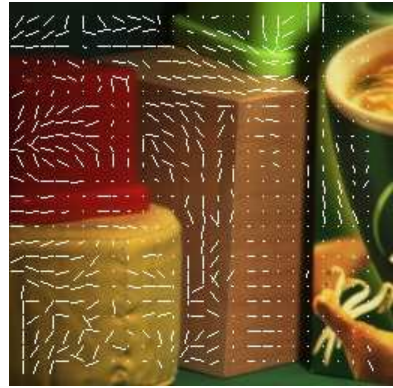

(b)

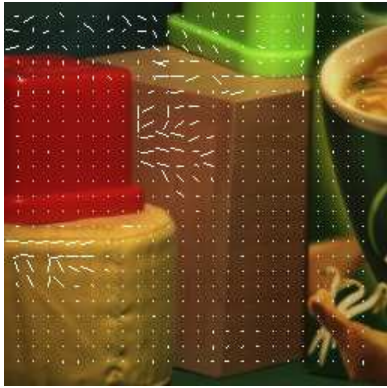

(c)

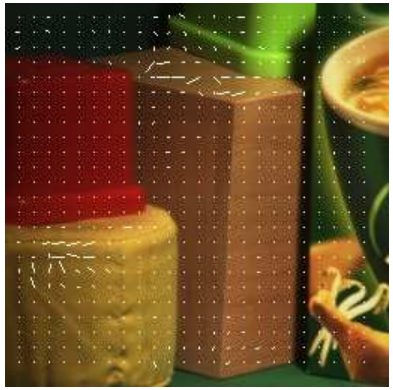

(d)

Fig. 5. a) frame 1 of object scene with fi lter size superimposed on it. b) $R G B$ gradient optical fbw c) shadow-shading invariant optical fbw and d) robust shadow-shading invariant optical fbw.

y-axis of Fig. 4a are depicted. Similarly to the shadow-shading-specular invariant optical flow is tested on a sequence with increasing achromaticity along the axes (see Fig. 4d,e,f.). The results show that robust invariant methods (red lines) outperform the standard photometric optical flow (blue lines). The gained robustness becomes apparent for the measurements around the instable region. Which are the black area for the shadow-shading invariant and the achromatic, grey area for the shadow-shading-specular invariant optical flow.

As an example of a real-world scene, multiple frames are taken from static objects while the light source position is changed. This results in a violation of the brightness constraint by changing shading and moving shadows. Since both the camera and the objects did not move the ground truth optical flow is zero. The violation of the brightness constraint disturbs the optical flow estimation based on the $R G B$ (Fig. 5b). The shadow-shading invariant optical flow estimation is much less disturbed by the violation of the brightness constrain(Fig. 5c). However it is still unstable around some of the edges. The robust shadow-shading invariant optical flow has the best results and is only unstable in low-gradient area's (Fig. 5d).

\section{Color Canny Edge Detection}

We illustrate the use of eigenvalue-based features by adapting the Canny edge detection algorithm to allow for vectorial input data. The algorithm consists of the following steps

1) Compute the spatial derivatives, $\mathbf{f}_{x}$, and combine them if desired into a quasi-invariant (Eq. 8 or Eq. 11).

2) Compute the maximum eigenvalue (Eq. 18) and its orientation (Eq. 19). 


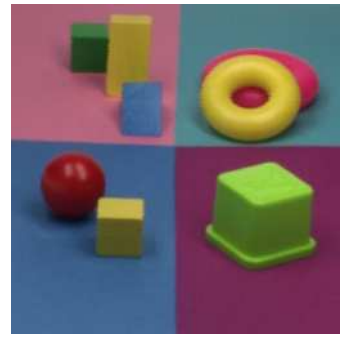

(a)

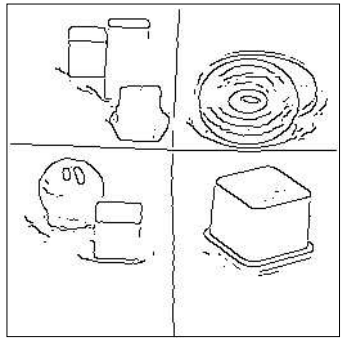

(b)

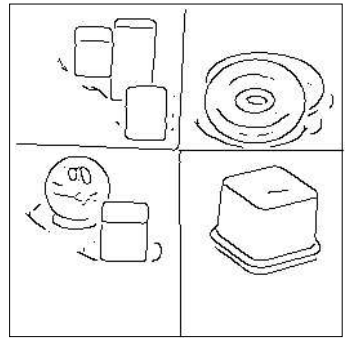

(c)

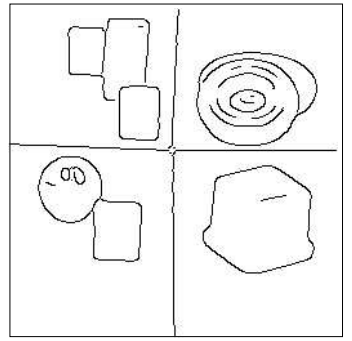

(d)

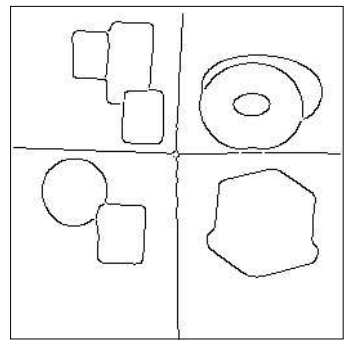

(e)

Fig. 6. a) input image with Canny edge detection based on successively b) luminance derivative c) RGB derivatives d) the shadow-shading quasi-invariant e) the shadow-shading-specular quasi-invariant.

3) Apply non-maximum suppression on $\lambda_{1}$ in the prominent direction.

In Fig. 6 the results of color Canny edge detection for several photometric quasi-invariants is shown. The results show that the luminance-based Canny, Fig. 6b, misses several edges which are correctly found by the $R G B$-based method, Fig. 6c. Also the removal of spurious edges by photometric invariance is demonstrated. In Fig. 6d the edge detection is robust to shadow and shading changes and only detects material and specular edges. In Fig. 6e only the material edges are depicted.

\section{Circular Object Detection}

The use of photometric invariant orientation and curvature estimation is demonstrated on a circle detection example. Other than the previous experiments these images have been recorded by the Nikon Coolpix 950, a commercial digital camera of average quality. The images have size 267x200 pixels with JPEG compression. The digitization was done in 8 bits per color.

Circular object recognition is complicated due to shadow, shading and specular events which influence the feature extraction. We apply the following algorithm for circle detection

1) Compute the spatial derivatives, $\mathbf{f}_{x}$, and combine them if desired into a quasi-invariant (Eq. 8 or Eq. 11).

2) Compute the local orientation, Eq. 19, and curvature, Eq. 23.

3) Compute the hough space ([1]), $H\left(R, x^{0}, y^{0}\right)$, where $R$ is the radius of the circle and $x^{0}$ and $y^{0}$ indicate the center of the circle. The computation of the orientation and curvature 


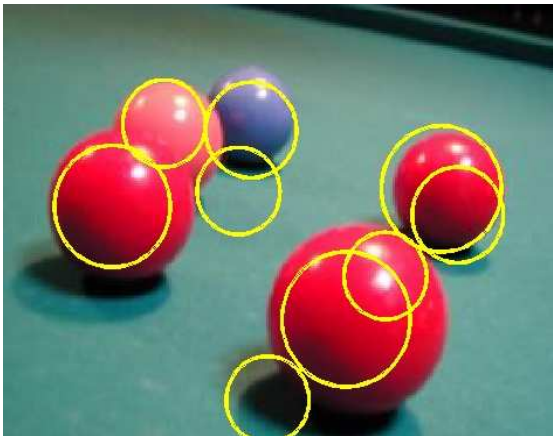

(a)

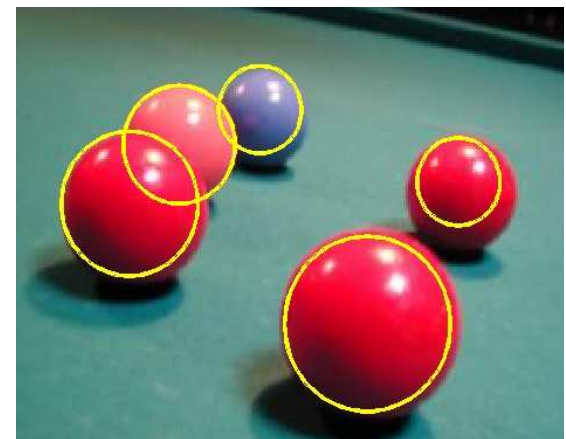

(b)

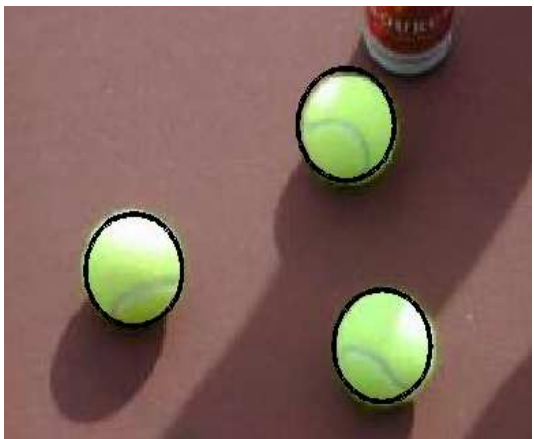

(c)

Fig. 7. a) detected circles based on luminance b) detected circles based on shadow-shading-specular quasi-invariant c) detected circles based on shadow-shading-specular quasi-invariant.

reduces the number of votes per pixel to one. Namely, for a pixel at position $\mathbf{x}=\left(x^{1}, y^{1}\right)$,

$$
\begin{aligned}
& R=\frac{1}{\kappa} \\
& x^{0}=x^{1}+\frac{1}{\kappa} \cos \theta \\
& y^{0}=y^{1}+\frac{1}{\kappa} \sin \theta
\end{aligned}
$$

Every pixel votes with its the derivative energy $\sqrt{\mathbf{f}_{x} \cdot \mathbf{f}_{x}}$.

4) Compute the maxima in the hough space. These maxima indicate the circle centers and the radii of the circle.

In Fig. 7 the results of the circle detection are given. The luminance-based circle detection is corrupted by the photometric variation in the image. Nine circles had to be detected before the five balls were detected. For the shadow-shading-specular quasi-invariant based method the five most prominent peaks in the hough space coincide with reasonable estimates of the radii and center points of the circles. Note that, although the recordings do not fulfill the assumptions on which the dichromatic model is based, such as white light source, saturated pixels and linear camera response, the invariants still improve performance by partially suppressing scene incidental events, such as shadows and specularities. In Fig. 7 an outdoor example with a shadow partially covering the objects is given.

\section{E. Local Color Symmetry Detector}

The applicability of the features derived from an adaptation of the structure tensor (section IV-B) is illustrated here for a symmetry detector. We apply the circle detector to an image 


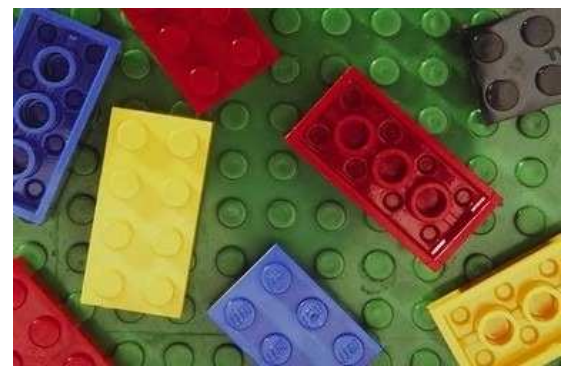

(a)

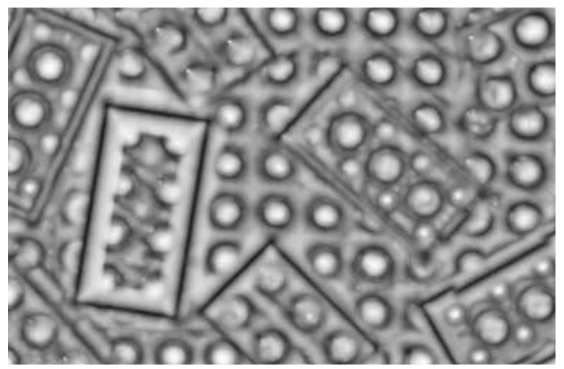

(b)

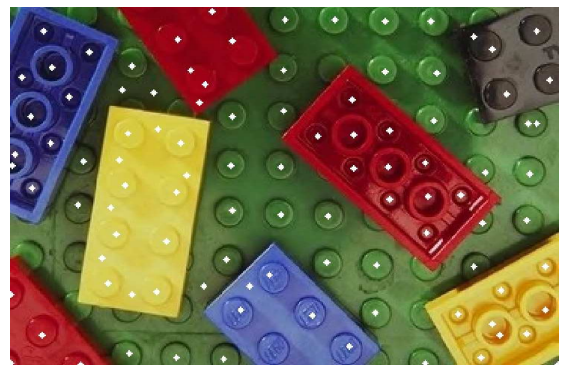

(c)

Fig. 8. a) input image b) the circularity coeffi cient $C$ c) the detected circles.

containing Lego-blocks (Fig. 8). Because we know that the color within the blocks remains the same, the circle detection is done on the shadow-shading-specular variant, $\mathbf{H}_{x}$ (Eq. 11). The shadow-shading-specular variant contains all the derivative energy except for the energy which can only be caused by a material edge. With the shadow-shading-specular variant the circular energy $\lambda_{1}$ and the starlike energy $\lambda_{2}$ are computed according to Eq. 22. Dividing the circular energy by the total energy yields a descriptor of local circularity (see Fig. 8b)

$$
C=\frac{\lambda_{1}}{\lambda_{1}+\lambda_{2}}
$$

The superimposed maxima of $C$, Fig. 8c, give good estimation of the circle centers.

\section{CONClusions}

In this paper we proposed a framework to combine tensor-based features and photometric invariance theory. The tensor basis of these features ensures that opposing vectors in different channels do not cancel out, but instead that they reinforce each other. To overcome the instability caused by transformation to an photometric full invariant, we propose an uncertainty measure to accompany the full invariant. This uncertainty measure is incorporated in the color tensor to generate robust photometric invariant features. Experiments show that the color based features outperform their luminance counterparts, that the quasi-invariants give stable detection, and that the robust invariants give better extraction results.

\section{REFERENCES}

[1] D. H. Ballard. Generalizing the Hough transform to detect arbitrary shapes. Pattern Recognition, 12(2):111-122, 1981. 
[2] J. Barron and R. Klette. Quantitative color optical fbw. In Int. Conf. on Pattern Recognition, pages 251-255, Vancouver, Canada, 2002.

[3] J. Bigun. Pattern recognition in images by symmetry and coordinate transformations. Computer Vision and Image Understanding, 68(3):290-307, 1997.

[4] J. Bigun, G. Granlund, and J. Wiklund. Multidimensional orientation estimation with applications to texture analysis and opitcal fbw. IEEE trans. on pattern analysis and machine intelligence, 13(8):775-790, 1991.

[5] Silvano Di Zenzo. Note: A note on the gradient of a multi-image. Computer Vision, Graphics, and Image Processing, 33(1):116-125, 1986.

[6] L. M. J. Florack, B. M. ter Haar Romeny, J. J. Koenderink, and M. A. Viergever. Scale and the differential structure of images. Image and Vision Computing, 10(6):376-388, July/August 1992.

[7] J.M. Geusebroek, R. van den Boomgaard, A.W.M. Smeulders, and H. Geerts. Color invariance. IEEE Trans. Pattern Analysis Machine Intell., 23(12):1338-1350, 2001.

[8] Th. Gevers. Color image invariant segmentation and retrieval. PhD thesis, University of Amsterdam, 1997.

[9] Th. Gevers and A. Smeulders. Color based object recognition. Pattern Recognition, 32:453-464, March 1999.

[10] Th. Gevers and H.M.G. Stokman. Classifi cation of color edges in videa into shadow-geometry, highlight, or material transitions. IEEE Trans. on Multimedia, 5(2):237-243, 2003.

[11] P. Golland and A. M. Bruckstein. Motion from color. Computer Vision and Image Understanding, 68(3):346-362, December 1997.

[12] O. Hansen and J. Bigun. Local symmetry modeling in multi-dimensional images. pattern Recognition Letters, 13:253-262, 1992.

[13] R.M. Haralick and L.G. Shapiro. Computer and Robot Vision, volume II. Addison-Wesley, 1992.

[14] C. Harris and M. Stephens. A combined corner and edge detector. In Proc. 4th Alvey Vision Conf., volume 15, pages 147-151, 1988.

[15] B. K. P. Horn and B. G. Schunk. Determing optical fbw. Artificial Intelligence, 17:185-203, 1981.

[16] M. Kass and A. Witkin. Analyzing oriented patterns. Computer Vision, Graphics, and Image Processing, 37:362-385, 1987.

[17] G.J. Klinker and S.A. Shafer. A physical approach to color image understanding. Int. Journal of Computer Vision, 4:7-38, 1990.

[18] D. Koubaroulis, J. Matas, and J. Kittler. Evaluating colour-based object recognition algorithms using the soil-47 database. In Asian Conference on Computer Vision, 2002.

[19] M.S. Lee and G. Medioni. Grouping into regions, curves, and junctions. Computer Vision Image Understanding, 76(1):5469, 1999.

[20] B. Lucas and T. Kanade. An iterative image registration technique with an application to stereo vision. In Proc. DARPA Image Understanding Workshop, pages 121-130, 1981.

[21] G. Sapiro and D. Ringach. Anisotropic diffusion of multivalued images with applications to color fi ltering. IEEE Trans. Image Processing, 5(11):1582-1586, Oct 1996.

[22] C. Schmid, R. Mohr, and C. Bauckhage. Evaluation of interest point detectors. International Journal of Computer Vision, 37(2):151-172, 2000.

[23] S.A. Shafer. Using color to seperate reftection components. COLOR research and application, 10(4):210-218, Winter 1985. 
[24] J. Shi and C. Tomasi. Good features to track. In IEEE conference on Computer Vision and Pattern Recognition, 1994.

[25] E. P. Simoncelli, E.H. Adelson, and D.J. Heeger. Probability distributions of optical fbw. In IEEE conference on Computer Vision and Pattern Recognition, pages 310-315, 1991.

[26] J. van de Weijer, Th. Gevers, and J.M. Geusebroek. Color edge detection by photometric quasi-invariants. In Int'l Conf. Computer Vision, pages 1520-1526, Nice, France, 2003.

[27] J. van de Weijer, L.J. van Vliet, P.W. Verbeek, and M. van Ginkel. Curvature estimation in oriented patterns using curvilinear models applied to gradient vector fi elds. IEEE Trans. Pattern Analysis and Machine Intelligence, 23(9):1035-1042, 2001. 